\title{
Nontrivial Solution of Fractional Differential System Involving Riemann-Stieltjes Integral Condition
}

\author{
Ge-Feng Yang \\ Cisco School of Informatics, Guangdong University of Foreign Studies, Guangzhou 510006, China
}

Correspondence should be addressed to Ge-Feng Yang, gefengyang_001@163.com

Received 14 September 2012; Accepted 25 October 2012

Academic Editor: Xinguang Zhang

Copyright (C) 2012 Ge-Feng Yang. This is an open access article distributed under the Creative Commons Attribution License, which permits unrestricted use, distribution, and reproduction in any medium, provided the original work is properly cited.

We study the existence and uniqueness of nontrivial solutions for a class of fractional differential system involving the Riemann-Stieltjes integral condition, by using the Leray-Schauder nonlinear alternative and the Banach contraction mapping principle, some sufficient conditions of the existence and uniqueness of a nontrivial solution of a system are obtained.

\section{Introduction}

HIV is a retrovirus that targets the $\mathrm{CD} 4^{+} \mathrm{T}$ lymphocytes, which are the most abundant white blood cells of the immune system. To this day, there have already been over 16 million people who died of AIDS. Although HIV infects other cells also, it wreaks the most havoc on the $\mathrm{CD}^{+} \mathrm{T}$ cells by causing their decline and destruction, thus decreasing the resistance of the immune system [1-3]. Mathematical models have been proven valuable in understanding the dynamics of HIV infection [4-6]. Perelson et al. [7, 8] developed a simple model for the primary infection with HIV. In this model, four categories of cells were defined: uninfected $\mathrm{CD}^{+}{ }^{+} \mathrm{T}$ cells, latently infected $\mathrm{CD} 4^{+} \mathrm{T}$ cells, productively infected $\mathrm{CD} 4^{+} \mathrm{T}$ cells, and virus population. And the following two equations describe the evolution of the system:

$$
\begin{gathered}
\frac{d x}{d t}=s-\mu x-\beta x y \\
\frac{d y}{d t}=\beta x y-v y,
\end{gathered}
$$


where all parameters and variables are nonnegative. $s$ is the assumed constant rate of the production of $\mathrm{CD}^{+} \mathrm{T}$-cells, $\mu$ is their per capita death rate, $\beta x y$ is the rate of infection of $\mathrm{CD} 4^{+} \mathrm{T}$-cells by virus, and $v y$ is the rate of disappearance of infected cells. Recently Arafa1 et al. introduced fractional order into a model of (HIV-1) infection of CD4 $4^{+} \mathrm{T}$ cells. The new system is described by the following set of FODEs of order $\alpha_{1}, \alpha_{2}, \alpha_{3}>0$ :

$$
\begin{gathered}
D^{\alpha_{1}}(T)=s-K V T-d T+b I, \\
D^{\alpha_{2}}(I)=K V T-(b+\delta) I, \\
D^{\alpha_{3}}(I)=N \delta I-c V .
\end{gathered}
$$

$T, I$, and $V$ denote the concentration of uninfected $\mathrm{CD}^{+} \mathrm{T}$ cells, infected CD4 ${ }^{+} \mathrm{T}$ cells, and free HIV virus particles in the blood, respectively. $\delta$ represents death rate of infected $\mathrm{T}$ cells and includes the possibility of death by the bursting of infected T cells, hence $\delta d$. The parameter $b$ is the rate at which infected cells return to uninfected class while $c$ is the death rate of virus and $N$ is the average number of viral particles produced by an infected cell.

Motivated by HIV model, in this paper, we consider the existence of nontrivial solution for fractional differential system

$$
\begin{gathered}
-\Phi_{\mathfrak{t}}^{\alpha} x(t)=\lambda f\left(t, x(t), \Phi_{\mathfrak{t}}^{\beta} x(t), y(t)\right), \quad-\Phi_{\mathfrak{t}}^{\gamma} y(t)=g(t, x(t)), \quad t \in(0,1), \\
\Phi_{\mathfrak{t}}^{\beta} x(0)=0, \quad \Phi_{\mathfrak{t}}^{\beta} x(1)=\int_{0}^{1} \Phi_{\mathfrak{t}}^{\beta} x(s) d A(s), \quad y(0)=0, \quad y(1)=\int_{0}^{1} y(s) d B(s),
\end{gathered}
$$

where $\lambda$ is a parameter, $1<\gamma<\alpha \leq 2,1<\alpha-\beta<\gamma, 0<\beta<1, \Phi_{\mathrm{t}}^{\alpha}$ is the standard Riemann-Liouville derivative. $\int_{0}^{1} \Phi_{\mathrm{t}}^{\beta} x(s) d A(s)$ denotes the Riemann-Stieltjes integral, and $A, B \in N B V([0,1])$ are functions of bounded variation.

In the recent years, there has been a significant development in fractional order differential equations involving fractional derivatives. For example, Ahmad and Nieto [9] considered a coupled system of nonlinear fractional differential equations with three-point boundary conditions

$$
\begin{gathered}
\Phi_{\mathrm{t}}^{\alpha} u(t)=f\left(t, v(t), \Phi_{\mathrm{t}}^{p} v(t)\right), \quad \Phi_{\mathrm{t}}^{\beta} v(t)=f\left(t, u(t), \Phi_{\mathrm{t}}^{q} u(t)\right), \quad t \in(0,1), \\
u(0)=0, \quad u(1)=\gamma u(\eta), \quad v(0)=0, \quad v(1)=\gamma v(\eta),
\end{gathered}
$$

where $\alpha, \beta, p, q, \gamma, \eta$ satisfy certain conditions. Applying the Schauder fixed point theorem, an existence result is proved provided that $f, g:[0,1] \times \mathbb{R} \times \mathbb{R} \rightarrow \mathbb{R}$ are given continuous functions and satisfy some growth conditions. For a detailed description of recent work on fractional differential equation, we refer the reader to some recent papers (see [10-17]).

The rest of the paper is organized as follows. Section 2 gives preliminaries and lemmas about fractional calculus. In Section 3, we present the main results and the proof of the results. In addition, an example is given to illustrate the application of the main results. 


\section{Preliminaries and Lemmas}

For the convenience of the reader, we present here some definitions from fractional calculus which are to be used in the later sections.

Definition 2.1 (see [18-20]). The Riemann-Liouville fractional integral of order $\alpha>0$ of a function $x:(0,+\infty) \rightarrow \mathbb{R}$ is given by

$$
I^{\alpha} x(t)=\frac{1}{\Gamma(\alpha)} \int_{0}^{t}(t-s)^{\alpha-1} x(s) d s
$$

provided that the right-hand side is pointwise defined on $(0,+\infty)$.

Definition 2.2 (see [18-20]). The Riemann-Liouville fractional derivative of order $\alpha>0$ of a function $x:(0,+\infty) \rightarrow \mathbb{R}$ is given by

$$
\Phi_{\mathfrak{t}}^{\alpha} x(t)=\frac{1}{\Gamma(n-\alpha)}\left(\frac{d}{d t}\right)^{n} \int_{0}^{t}(t-s)^{n-\alpha-1} x(s) d s,
$$

where $n=[\alpha]+1,[\alpha]$ denotes the integer part of number $\alpha$, provided that the right-hand side is pointwise defined on $(0,+\infty)$.

Lemma 2.3 (see [18-20]). (1) If $x \in L(0,1), v>\sigma>0$, then

$$
I^{v} I^{\sigma} x(t)=I^{v+\sigma} x(t), \quad \Phi_{\mathfrak{t}}^{\sigma} I^{v} x(t)=I^{v-\sigma} x(t), \quad \Phi_{\mathfrak{t}}^{\sigma} I^{\sigma} x(t)=x(t) .
$$

(2) If $v>0, \sigma>0$, then

$$
\Phi_{\mathbf{t}}^{v} t^{\sigma-1}=\frac{\Gamma(\sigma)}{\Gamma(\sigma-v)} t^{\sigma-v-1}
$$

Lemma 2.4 (see [18-20]). Assume that $x \in L^{1}(0,1)$ with a fractional derivative of order $\alpha>0$ that belongs to $L^{1}(0,1)$. Then

$$
I^{\alpha} \boldsymbol{\Phi}_{\mathfrak{t}}^{\alpha} x(t)=x(t)+c_{1} t^{\alpha-1}+c_{2} t^{\alpha-2}+\cdots+c_{n} t^{\alpha-n}
$$

where $c_{i} \in \mathbb{R}(i=1,2, \ldots, n), n$ is the smallest integer greater than or equal to $\alpha$.

Let $x(t)=I^{\beta} v(t), v(t) \in C[0,1]$; by standard discussion, we easily reduce the system (1.1) to the following modified problems:

$$
\begin{aligned}
& -\Phi_{\mathrm{t}}^{\alpha-\beta} v(t)=\lambda f\left(t, I^{\beta} v(t), v(t), y(t)\right), \quad-\Phi_{\mathrm{t}}^{\gamma} y(t)=g\left(t, I^{\beta} v(t)\right), \quad t \in(0,1), \\
& v(0)=0, \quad v(1)=\int_{0}^{1} v(s) d A(s), \quad y(0)=0, \quad y(1)=\int_{0}^{1} y(s) d B(s),
\end{aligned}
$$

and the system (2.6) is equivalent to the system (1.1). 
Lemma 2.5 (see [21]). Let $h \in L^{1}(0,1)$, if $1<\alpha-\beta, \gamma \leq 2$, then the unique solution of the linear problems

$$
\begin{gathered}
-\Phi_{\mathrm{t}}^{\alpha-\beta} v(t)=h(t), \quad t \in(0,1), \\
v(0)=0, \quad v(1)=0, \\
-\Phi_{\mathrm{t}}^{\gamma} y(t)=h(t), \quad t \in(0,1), \\
y(0)=0, \quad y(1)=0,
\end{gathered}
$$

is

$$
v(t)=\int_{0}^{1} K_{1}(t, s) h(s) d s, \quad y(t)=\int_{0}^{1} K_{2}(t, s) h(s) d s,
$$

respectively, where

$$
\begin{gathered}
K_{1}(t, s)=\frac{1}{\Gamma(\alpha-\beta)} \begin{cases}{[t(1-s)]^{\alpha-\beta-1},} & 0 \leq t \leq s \leq 1, \\
{[t(1-s)]^{\alpha-\beta-1}-(t-s)^{\alpha-\beta-1},} & 0 \leq s \leq t \leq 1 .\end{cases} \\
K_{2}(t, s)=\frac{1}{\Gamma(\gamma)} \begin{cases}{[t(1-s)]^{\gamma-1},} & 0 \leq t \leq s \leq 1, \\
{[t(1-s)]^{\gamma-1}-(t-s)^{\gamma-1},} & 0 \leq s \leq t \leq 1,\end{cases}
\end{gathered}
$$

are the Green functions of the boundary value problems (2.7).

By Lemma 2.4, the unique solution of the problem

$$
\begin{gathered}
\Phi_{\mathrm{t}}^{\alpha-\beta} v(t)=0, \quad 0<t<1, \\
v(0)=0, \quad v(1)=1,
\end{gathered}
$$

is $t^{\alpha-\beta-1}$. Let

$$
\mathcal{C}=\int_{0}^{1} t^{\alpha-\beta-1} d A(t), \quad B=\int_{0}^{1} t^{\gamma-1} d B(t)
$$

and define

$$
\mathcal{G}_{A}(s)=\int_{0}^{1} K_{1}(t, s) d A(t), \quad \mathcal{G}_{B}(s)=\int_{0}^{1} K_{2}(t, s) d B(t) .
$$


As in $[14]$, if $\mathcal{C} \neq 0, B \neq 0$, we can get that the Green function for the following nonlocal system

$$
\begin{gathered}
-\Phi_{\mathrm{t}}^{\alpha-\beta} v(t)=h(t), \quad t \in(0,1), \\
v(0)=0, \quad v(1)=\int_{0}^{1} v(s) d A(s), \\
-\Phi_{\mathrm{t}}^{\gamma} y(t)=h(t), \quad t \in(0,1), \\
y(0)=0, \quad y(1)=\int_{0}^{1} y(s) d B(s),
\end{gathered}
$$

are given by, respectively,

$$
G(t, s)=\frac{t^{\alpha-\beta-1}}{1-\mathcal{C}} \mathcal{G}_{A}(s)+K_{1}(t, s), \quad H(t, s)=\frac{t^{\gamma-1}}{1-B} \mathcal{G}_{B}(s)+K_{2}(t, s)
$$

Clearly, $G(t, s), H(t, s)$ are continuous on $[0,1] \times[0,1]$; thus there exist positive constants $m, n$ such that

$$
|G(t, s)| \leq m, \quad|H(t, s)| \leq n .
$$

It is well known that $(v, y)$ is a solution of the system (2.6) if and only if $(v, y) \in$ $C[0,1] \times C[0,1]$ is a solution of the following nonlinear integral equation system:

$$
\begin{gathered}
v(t)=\lambda \int_{0}^{1} G(t, s) f\left(s, I^{\beta} v(s), v(s), y(s)\right) d s, \\
y(t)=\int_{0}^{1} H(t, s) g\left(s, I^{\beta} v(s)\right) d s .
\end{gathered}
$$

Obviously, the system (2.16) is equivalent to the following integral equation:

$$
v(t)=\lambda \int_{0}^{1} G(t, s) f\left(s, I^{\beta} v(s), v(s), \int_{0}^{1} H(s, \tau) g\left(\tau, I^{\beta} v(\tau)\right) d \tau\right) d s .
$$

Lemma 2.6 (see [22]). Let $X$ be a real Banach space and $\Omega$ a bounded open subset of $X$, where $\theta \in \Omega$; $T: \bar{\Omega} \rightarrow X$ is a completely continuous operator. Then, either there exists $x \in \partial \Omega, \lambda>1$ such that $T(x)=\lambda x$ or there exists a fixed point $x^{*} \in \bar{\Omega}$.

\section{Main Results}

The following definition introduces the Carathèodory conditions imposed on a map $f$. 
Definition 3.1. Let $\widehat{z}=\left(z_{1}, z_{2}, \ldots, z_{n}\right)$. A map $f:[0,1] \times \mathbb{R}^{n},(t, \widehat{z}) \mapsto f(t, \widehat{z})$ is said to satisfy the Carathéodory conditions if the following conditions hold:

(i) for each $\widehat{z} \in \mathbb{R}^{n}$, the mapping $t \mapsto f(t, \widehat{z})$ is Lebesgue measurable;

(ii) for a.e. $t \in[0,1]$, the mapping $\widehat{z} \mapsto f(t, \widehat{z})$ is continuous on $\mathbb{R}$.

Throughout the paper we always assume the following conditions hold.

(H0) $A, B$ are functions of bounded variation such that $\mathcal{C}, B \neq 1$, where $\mathcal{C}, \mathbb{B}$ are defined by $(2.11)$.

(H1) $f:[0,1] \times \mathbb{R}^{3} \rightarrow \mathbb{R}$ and $g:[0,1] \times \mathbb{R} \rightarrow \mathbb{R}$ satisfy the Carathéodory condition.

Theorem 3.2. Suppose that $(H 0)-(H 1)$ hold. If $f(t, 0,0,0) \not \equiv 0$, and there exist nonnegative functions $p_{i}(i=1,2,3), q, r \in L^{1}[0,1]$ such that

$$
\begin{gathered}
\left|f\left(t, x_{1}, x_{2}, x_{3}\right)\right| \leq p_{1}(t)\left|x_{1}\right|+p_{2}(t)\left|x_{2}\right|+p_{3}(t)\left|x_{3}\right|+q(t), \quad \text { a.e. } t \in[0,1], \\
|g(t, y)| \leq r(t)|y|, \quad \text { a.e. } t \in[0,1] .
\end{gathered}
$$

In addition, there exists $t_{0} \in[0,1]$ such that $p_{i_{0}}\left(t_{0}\right) \neq 0$ for some $i_{0} \in\{1,2,3\}$. Then there exists a constant $\lambda^{*}>0$, such that, for any $0<\lambda \leq \lambda^{*}$, the system (1.1) has at least one nontrivial solution $\left(x^{*}, y^{*}\right) \in C[0,1] \times C[0,1]$.

Proof. Let $X=C[0,1]$ be endowed with the ordering $x \leq y$ if $x(t) \leq y(t)$ for all $t \in[0,1]$, and $\|u\|=\max _{t \in[0,1]}|u(t)|$ is defined as usual by maximum norm. Clearly, it follows that $(X,\|\cdot\|)$ is a Banach space. By (2.17) and (2.6), problem (1.1) has a solution $\left(x^{*}, y^{*}\right) \in C[0,1] \times C[0,1]$ if and only if $v^{*}=\Phi_{t}^{\beta} x^{*}$ solves the following operator equation:

$$
(T v)(t)=\lambda \int_{0}^{1} G(t, s) f\left(s, I^{\beta} v(s), v(s), \int_{0}^{1} H(s, \tau) g\left(\tau, I^{\beta} v(\tau)\right) d \tau\right) d s,
$$

in $X$. So we only need to seek a fixed point of $T$ in $X$. By Ascoli-Arzela Theorem, it is obvious that the operator $T: X \rightarrow X$ is a completely continuous operator.

Since $f(t, 0,0,0) \not \equiv 0$, and $f(t, 0,0,0) \leq q(t)$ a.e. $t \in[0,1]$, we know $\int_{0}^{1} q(t) d t>0$. On the other hand, by $p_{i_{0}}\left(t_{0}\right) \neq 0$ for some $i_{0} \in\{1,2,3\}$, we have

$$
\int_{0}^{1}\left(p_{1}(t)+p_{2}(t)+p_{3}(t)\right) d t>0
$$

Let

$$
R=\frac{\int_{0}^{1} q(s) d s}{\left(1 / \Gamma(\beta)+1+(n / \Gamma(\beta)) \int_{0}^{1} r(s) d s\right) \int_{0}^{1}\left(p_{1}(s)+p_{2}(s)+p_{3}(s)\right) d s},
$$

where $n$ is defined by (2.15). Define the set

$$
\Omega=\{v \in C[0,1]:\|v\| \leq R\} .
$$


Suppose $v \in \partial \Omega, \mu>1$ such that $T v=\mu v$. Then, for any $v \in \partial \Omega$, and noticing that

$$
\left|I^{\beta} v(\tau)\right|=\frac{1}{\Gamma(\beta)} \int_{0}^{1}(t-s)^{\beta-1} v(s) d s \leq \frac{\|v\|}{\Gamma(\beta)},
$$

we have

$$
\begin{aligned}
\|T v\|= & \max _{t \in[0,1]}|(T v)(t)| \leq m \lambda \int_{0}^{1}\left|f\left(s, I^{\beta} v(s), v(s), \int_{0}^{1} H(s, \tau) g\left(\tau, I^{\beta} v(\tau)\right) d \tau\right)\right| d s \\
\leq & m \lambda \int_{0}^{1}\left[p_{1}(t)\left|I^{\beta} v(s)\right|+p_{2}(t)|v(s)|+p_{3}(t)\left|\int_{0}^{1} H(s, \tau) g\left(\tau, I^{\beta} v(\tau)\right) d \tau\right|+q(s)\right] d s \\
\leq & m \lambda \int_{0}^{1}\left[p_{1}(t)\left|I^{\beta} v(s)\right|+p_{2}(t)|v(s)|+n p_{3}(t)\left|\int_{0}^{1} r(\tau) I^{\beta} v(\tau) d \tau\right|+q(s)\right] d s \\
\leq & m \lambda \int_{0}^{1}\left[\frac{p_{1}(t)\|v\|}{\Gamma(\beta)}+p_{2}(t)\|v\|+\frac{n p_{3}(t)\|v\|}{\Gamma(\beta)} \int_{0}^{1} r(\tau) d \tau+q(s)\right] d s \\
\leq & m \lambda\left(\frac{1}{\Gamma(\beta)}+1+\frac{n}{\Gamma(\beta)} \int_{0}^{1} r(s) d s\right) \int_{0}^{1}\left(p_{1}(s)+p_{2}(s)+p_{3}(s)\right) d s\|v\| \\
& +m \lambda \int_{0}^{1} q(s) d s .
\end{aligned}
$$

Thus we take

$$
\lambda^{*}=\frac{1}{2}\left\{\left[m\left(\frac{1}{\Gamma(\beta)}+1+\frac{n}{\Gamma(\beta)} \int_{0}^{1} r(s) d s\right)\right] \int_{0}^{1}\left(p_{1}(s)+p_{2}(s)+p_{3}(s)\right) d s\right\}^{-1}
$$

Then for any $0<\lambda \leq \lambda^{*}$ and $v \in \partial \Omega$, by (3.7), one has

$$
\begin{aligned}
\mu\|v\| & \leq m \lambda\left(\frac{1}{\Gamma(\beta)}+1+\frac{n}{\Gamma(\beta)} \int_{0}^{1} r(s) d s\right) \int_{0}^{1}\left(p_{1}(s)+p_{2}(s)+p_{3}(s)\right) d s\|v\|+m \lambda \int_{0}^{1} q(s) d s \\
& \leq \frac{1}{2}\|v\|+\frac{\int_{0}^{1} q(s) d s}{2\left(1 / \Gamma(\beta)+1+(n / \Gamma(\beta)) \int_{0}^{1} r(s) d s\right) \int_{0}^{1}\left(p_{1}(s)+p_{2}(s)+p_{3}(s)\right) d s} .
\end{aligned}
$$

Consequently,

$$
\mu \leq \frac{1}{2}+\frac{1}{2}=1
$$


This contradicts $\mu>1$, by Lemma 2.6, $T$ has a fixed point $v^{*} \in \bar{\Omega}$. Since $f(t, 0,0,0) \not \equiv 0$, then $v^{*} \not \equiv 0$, Thus let

$$
x^{*}=I^{\beta} v^{*}, \quad y^{*}=\int_{0}^{1} H(t, s) g\left(s, x^{*}(s)\right) d s,
$$

and then the system (1.1) has at least one nontrivial solution $\left(x^{*}, y^{*}\right) \in C[0,1] \times C[0,1]$ for any $0<\lambda \leq \lambda^{*}$. This completes the proof of Theorem 3.2.

Theorem 3.3. Suppose that (H0)-(H1) hold. If $f(t, 0,0,0) \not \equiv 0$, and there exist nonnegative functions $p_{i}(i=1,2,3), r \in L^{1}[0,1]$ such that

$$
\begin{gathered}
\left|f\left(t, x_{1}, x_{2}, x_{3}\right)-f\left(t, y_{1}, y_{2}, y_{3}\right)\right| \leq p_{1}(t)\left|x_{1}-y_{1}\right|+p_{2}(t)\left|x_{2}-y_{2}\right|+p_{3}(t)\left|x_{3}-y_{3}\right|, \quad \text { a.e. } t \in[0,1] \\
\left|g\left(t, y_{1}\right)-g\left(t, y_{2}\right)\right| \leq r(t)\left|y_{1}-y_{2}\right|, \quad \text { a.e. } t \in[0,1] .
\end{gathered}
$$

In addition, there exists $t_{0} \in[0,1]$ such that $p_{i_{0}}\left(t_{0}\right) \neq 0$ for some $i_{0} \in\{1,2,3\}$. Then there exists a constant $\lambda^{*}>0$, such that, for any $0<\lambda \leq \lambda^{*}$, the system (1.1) has unique nontrivial solution $\left(x^{*}, y^{*}\right) \in C[0,1] \times C[0,1]$.

Proof. Let $T$ be given in Theorem 3.2; we will show that $T$ is a contraction. In fact,

$$
\begin{aligned}
&\left\|T v_{1}-T v_{2}\right\| \leq m \lambda \int_{0}^{1} \mid f\left(s, I^{\beta} v_{1}(s), v_{1}(s), \int_{0}^{1} H(s, \tau) g\left(\tau, I^{\beta} v_{1}(\tau)\right) d \tau\right) \quad-f\left(s, I^{\beta} v_{2}(s), v_{2}(s), \int_{0}^{1} H(s, \tau) g\left(\tau, I^{\beta} v_{2}(\tau)\right) d \tau\right) \mid d s \\
& \leq m \lambda \int_{0}^{1}\left[p_{1}(t)\left|I^{\beta}\left(v_{1}(s)-v_{2}(s)\right)\right|+p_{2}(t)\left|v_{1}(s)-v_{2}(s)\right|\right. \\
&\left.\quad+n p_{3}(t) \int_{0}^{1} r(\tau)\left|I^{\beta}\left(v_{1}(\tau)-v_{2}(\tau)\right)\right| d \tau\right] d s \\
& \leq m \lambda \int_{0}^{1}\left[\frac{p_{1}(t)\left\|v_{1}-v_{2}\right\|}{\Gamma(\beta)}+p_{2}(t)\left\|v_{1}-v_{2}\right\|+\frac{n p_{3}(t)}{\Gamma(\beta)} \int_{0}^{1} r(\tau) d \tau\left\|v_{1}-v_{2}\right\|\right] d s \\
& \leq m \lambda\left(\frac{1}{\Gamma(\beta)}+1+\frac{n}{\Gamma(\beta)} \int_{0}^{1} r(s) d s\right) \int_{0}^{1}\left(p_{1}(s)+p_{2}(s)+p_{3}(s)\right) d s\left\|v_{1}-v_{2}\right\| .
\end{aligned}
$$

If we choose

$$
\lambda^{*}=\frac{1}{2}\left\{m\left(\frac{1}{\Gamma(\beta)}+1+\frac{n}{\Gamma(\beta)} \int_{0}^{1} r(s) d s\right) \int_{0}^{1}\left(p_{1}(s)+p_{2}(s)+p_{3}(s)\right) d s\right\}^{-1} .
$$


Then by (3.13) and (3.14), we have

$$
\left\|T v_{1}-T v_{2}\right\| \leq \frac{1}{2}\left\|v_{1}-v_{2}\right\|
$$

which implies that $T$ is indeed a contraction. Finally, we use the Banach fixed point theorem to deduce the existence of a unique solution to the system (1.1).

Corollary 3.4. Suppose that $(H 0)-(H 1)$ hold. If $f(t, 0,0,0) \not \equiv 0$,

$$
\limsup _{\left|x_{1}\right|+\left|x_{2}\right|+\left|x_{3}\right| \rightarrow+\infty} \max _{t \in[0,1]} \frac{f\left(t, x_{1}, x_{2}, x_{3}\right)}{\left|x_{1}\right|+\left|x_{2}\right|+\left|x_{3}\right|}<+\infty
$$

In addition, there exists a nonnegative function $r \in L^{1}[0,1]$ such that $g$ satisfies

$$
|g(t, y)| \leq r(t)|y|, \quad \text { a.e. } t \in[0,1]
$$

Then there exists a constant $\lambda^{*}>0$, such that, for any $0<\lambda \leq \lambda^{*}$, the system (1.1) has at least one nontrivial solution $\left(x^{*}, y^{*}\right) \in C[0,1] \times C[0,1]$.

Proof. We prove $f$ satisfies the conditions of Theorem 3.2. Let

$$
\mathfrak{W}=\limsup _{\left|x_{1}\right|+\left|x_{2}\right|+\left|x_{3}\right| \rightarrow+\infty} \max _{t \in[0,1]} \frac{f\left(t, x_{1}, x_{2}, x_{3}\right)}{\left|x_{1}\right|+\left|x_{2}\right|+\left|x_{3}\right|}
$$

and choose $\varepsilon>0$ such that $\mathfrak{W}+1-\varepsilon>0$. By (3.16), there exists $N>0$ such that

$$
\left|f\left(t, x_{1}, x_{2}, x_{3}\right)\right| \leq(\mathfrak{W}+1-\varepsilon)\left(\left|x_{1}\right|+\left|x_{2}\right|+\left|x_{3}\right|\right), \quad\left|x_{1}\right|+\left|x_{2}\right|+\left|x_{3}\right|>N, t \in[0,1] .
$$

Take $\mathbb{M}=\max _{t \in[0,1] / \Omega,\left|x_{1}\right|+\left|x_{2}\right|+\left|x_{3}\right| \leq N},\left|f\left(t, x_{1}, x_{2}, x_{3}\right)\right|$, and the measure of $\Omega$ is 0 . Thus for any $\left(t, x, x_{2}, x_{3}\right) \in[0,1] \times \mathbb{R}^{3}$, we have

$$
\left|f\left(t, x_{1}, x_{2}, x_{3}\right)\right| \leq(\mathfrak{W}+1-\varepsilon)\left(\left|x_{1}\right|+\left|x_{2}\right|+\left|x_{3}\right|\right)+\mathbb{M} \text {, a.e. } t \in[0,1] .
$$

From Theorem 3.2 we know the system (1.1) has at least one nontrivial solution.

Example 3.5. Consider the following fractional differential system:

$$
\begin{gathered}
-\Phi_{\mathfrak{t}}^{3 / 2} x=\lambda\left[\left(t^{2}+\sin t\right) x-t^{1 / 2} \cos ^{2}\left(\Phi_{\mathfrak{t}}^{3 / 8} x\right)+\frac{1}{\sqrt[3]{t}} \sin y+\frac{1}{\sqrt{1-t}}+2\right], \\
-\Phi_{\mathfrak{t}}^{5 / 4} y=(t \cos t+\ln (t+1)) x, \\
\Phi_{\mathfrak{t}}^{3 / 8} x(0)=0, \quad \Phi_{\mathfrak{t}}^{3 / 8} x(1)=\int_{0}^{1} \Phi_{\mathfrak{t}}^{3 / 8} x(t) d A(t), \quad y(0)=0, \quad y(1)=\int_{0}^{1} y(t) d B(t),
\end{gathered}
$$


where

$$
A(t)=\left\{\begin{array}{ll}
0, & t \in\left[0, \frac{1}{2}\right), \\
\frac{3}{2}, & t \in\left[\frac{1}{2}, \frac{3}{4}\right), \\
1, & t \in\left[\frac{3}{4}, 1\right],
\end{array} \quad B(t)= \begin{cases}0, & t \in\left[0, \frac{1}{4}\right), \\
1, & t \in\left[\frac{1}{4}, \frac{3}{4}\right), \\
2, & t \in\left[\frac{3}{4}, 1\right] .\end{cases}\right.
$$

Then the system (3.21) is equivalent to the following 4-point BVP with coefficients of both signs

$$
\begin{gathered}
-\Phi_{\mathfrak{t}}^{3 / 2} x=\lambda\left[\left(t^{2}+\sin t\right) x-t^{1 / 2} \cos ^{2}\left(\Phi_{\mathfrak{t}}^{3 / 8} x\right)+\frac{1}{\sqrt[3]{t}} \sin y+\frac{1}{\sqrt{1-t}}+2\right], \\
-\Phi_{\mathfrak{t}}^{5 / 4} y=(t \cos t+\ln (t+1)) x, \\
\Phi_{\mathfrak{t}}^{3 / 8} x(0)=0, \quad \Phi_{\mathfrak{t}}^{3 / 8} x(1)=\frac{3}{2} \Phi_{\mathfrak{t}}^{3 / 8}\left(\frac{1}{2}\right)-\frac{1}{2} \Phi_{\mathfrak{t}}^{3 / 8}\left(\frac{3}{4}\right), \\
y(0)=0, \quad y(1)=y\left(\frac{1}{4}\right)+y\left(\frac{3}{4}\right) .
\end{gathered}
$$

Clearly, (H0) holds. Let

$$
f\left(t, x_{1}, x_{2}, x_{3}\right)=\left(t^{2}+\sin t\right) x_{1}-t^{1 / 2} \cos ^{2} x_{2}+\frac{1}{\sqrt[3]{t}} \sin x_{3}+\frac{1}{\sqrt{1-t}}+2, \quad g(t, y)=(t \cos t+\ln (t+1)) y .
$$

Then (H1) also is satisfied.

On the other hand, we have

$$
\begin{gathered}
\left|f\left(t, x_{1}, x_{2}, x_{3}\right)\right| \leq\left(t^{2}+\sin t\right)\left|x_{1}\right|+t^{1 / 2}\left|x_{2}\right|+\frac{1}{\sqrt[3]{t}}\left|x_{3}\right|+\frac{1}{\sqrt{1-t}}+2, \\
|g(t, y)| \leq(t \cos t+\ln (t+1))|y|
\end{gathered}
$$

and $f(t, 0,0,0)=1 / \sqrt{1-t}+2 \not \equiv 0$, which imply all conditions of Theorem 3.2 are satisfied, by Theorem 3.2, there exists a constant $\lambda^{*}>0$, such that for any $0<\lambda \leq \lambda^{*}$, the system (3.21) has at least one nontrivial solution $\left(x^{*}, y^{*}\right) \in C[0,1] \times C[0,1]$.

\section{References}

[1] P. W. Nelson and A. S. Perelson, "Mathematical analysis of delay differential equation models of HIV1 infection," Mathematical Biosciences, vol. 179, no. 1, pp. 73-94, 2002.

[2] L. Wang and M. Y. Li, "Mathematical analysis of the global dynamics of a model for HIV infection of CD4 ${ }^{+}$T cells," Mathematical Biosciences, vol. 200, no. 1, pp. 44-57, 2006. 
[3] R. V. Culshaw and S. Ruan, "A delay-differential equation model of HIV infection of CD4+ T-cells," Mathematical Biosciences, vol. 165, pp. 27-39, 2000.

[4] T. C. Henry and Y. M. W. Frederic, "On the behavior of solutions in viral dynamical models," Biosystems, vol. 73, pp. 157-161, 2004.

[5] M. Merdan and T. Y. Khan, "Homotopy perturbation method for solving viral dynamical model," Fen Bilimleri Dergisi, vol. 31, pp. 65-77, 2010.

[6] L. M. Petrovic, D. T. Spasic, and T. M. Atanackovic, "On a mathematical model of a human root dentin," Dental Materials, vol. 21, pp. 125-128, 2005.

[7] A. S. Perelson, "Modeling the interaction of the immune system with HIV," in Mathematical and Statistical Approaches to AIDS Epidemiology, vol. 83 of Lecture Notes in Biomathematics, pp. 350-370, Springer, Berlin, Germany, 1989.

[8] A. S. Perelson, D. E. Kirschner, and R. D. Boer, "Dynamics of HIV infection of CD4+ T cells," Mathematical Biosciences, vol. 114, pp. 81-125, 1993.

[9] B. Ahmad and J. J. Nieto, "Existence results for a coupled system of nonlinear fractional differential equations with three-point boundary conditions," Computers $\mathcal{E}$ Mathematics with Applications, vol. 58, no. 9, pp. 1838-1843, 2009.

[10] X. Zhang, L. Liu, and Y. Wu, "Multiple positive solutions of a singular fractional differential equation with negatively perturbed term," Mathematical and Computer Modelling, vol. 55, no. 3-4, pp. 1263-1274, 2012.

[11] C. S. Goodrich, "Existence and uniqueness of solutions to a fractional difference equation with nonlocal conditions," Computers \& Mathematics with Applications, vol. 61, no. 2, pp. 191-202, 2011.

[12] C.S. Goodrich, "Positive solutions to boundary value problems with nonlinear boundary conditions," Nonlinear Analysis. Theory, Methods E Applications, vol. 75, no. 1, pp. 417-432, 2012.

[13] B. Ahmad and J. J. Nieto, "Existence results for higher order fractional differential inclusions with nonlocal boundary conditions," Nonlinear Studies, vol. 17, no. 2, pp. 131-138, 2010.

[14] X. Zhang and Y. Han, "Existence and uniqueness of positive solutions for higher order nonlocal fractional differential equations," Applied Mathematics Letters, vol. 25, no. 3, pp. 555-560, 2012.

[15] W. Jiang, "The existence of solutions to boundary value problems of fractional differential equations at resonance," Nonlinear Analysis. Theory, Methods E Applications, vol. 74, no. 5, pp. 1987-1994, 2011.

[16] X. Zhang, L. Liu, and Y. Wu, "The eigenvalue problem for a singular higher order fractional differential equation involving fractional derivatives," Applied Mathematics and Computation, vol. 218, no. 17, pp. 8526-8536, 2012.

[17] X. Zhang, L. Liu, and Y. Wu, "Existence results for multiple positive solutions of nonlinear higher order perturbed fractional differential equations with derivatives," Applied Mathematics and Computation, vol. 219, no. 4, pp. 1420-1433, 2012.

[18] I. Podlubny, Fractional Differential Equations, vol. 198 of Mathematics in Science and Engineering, Academic Press, San Diego, Calif, USA, 1999.

[19] K. S. Miller and B. Ross, An Introduction to the Fractional Calculus and Fractional Differential Equations, A Wiley-Interscience Publication, John Wiley \& Sons, New York, NY, USA, 1993.

[20] A. A. Kilbas, H. M. Srivastava, and J. J. Trujillo, Theory and Applications of Fractional Differential Equations, vol. 204, Elsevier Science B.V., Amsterdam, The Netherlands, 2006.

[21] Z. Bai and H. Lü, "Positive solutions for boundary value problem of nonlinear fractional differential equation," Journal of Mathematical Analysis and Applications, vol. 311, no. 2, pp. 495-505, 2005.

[22] K. Deimling, Nonlinear Functional Analysis, Springer, Berlin, Germany, 1985. 


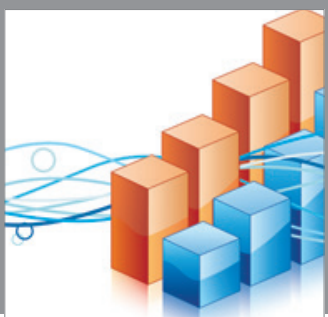

Advances in

Operations Research

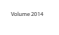

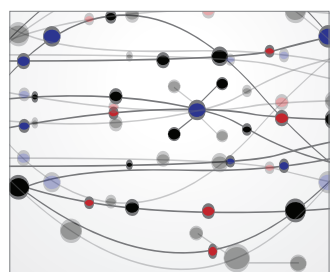

\section{The Scientific} World Journal
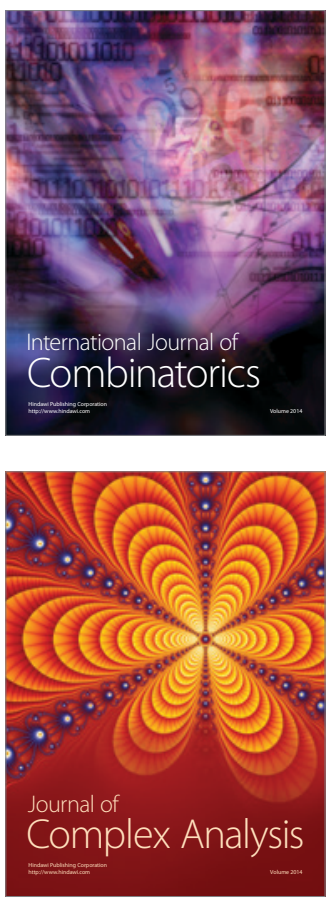

International Journal of

Mathematics and

Mathematical

Sciences
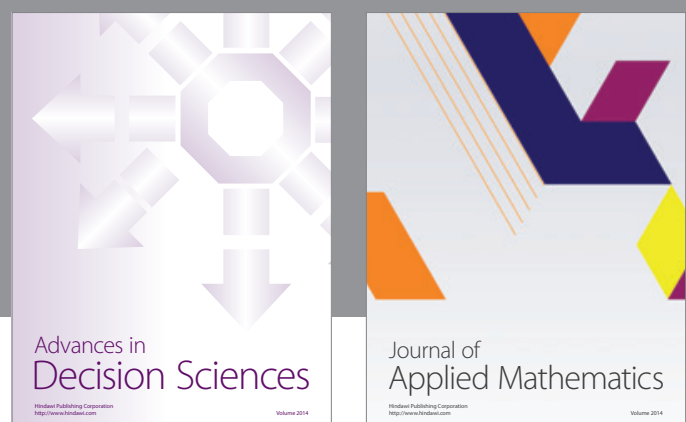

Journal of

Applied Mathematics
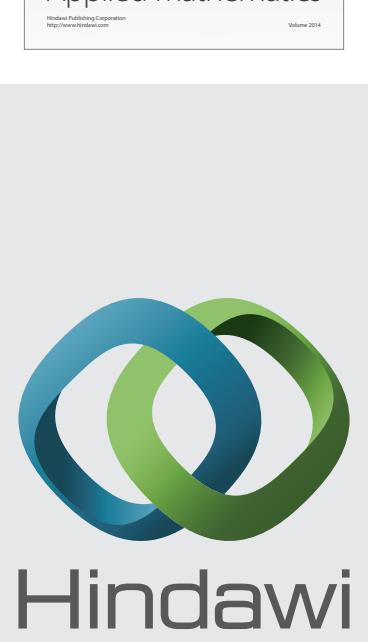

Submit your manuscripts at http://www.hindawi.com
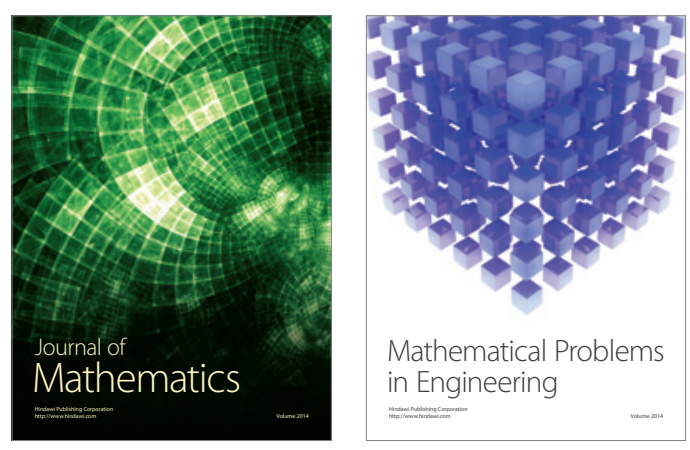

Mathematical Problems in Engineering
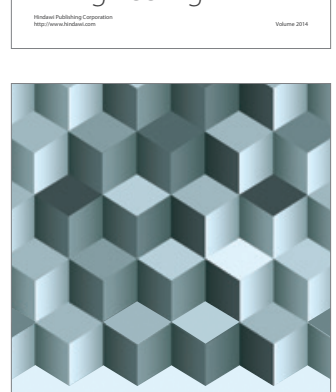

Journal of

Function Spaces
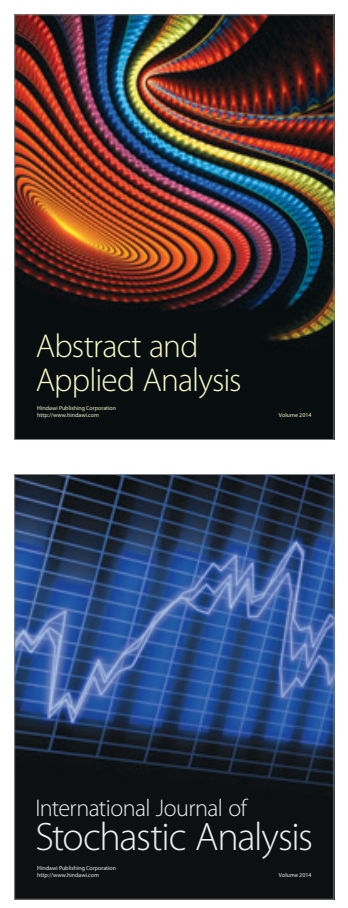

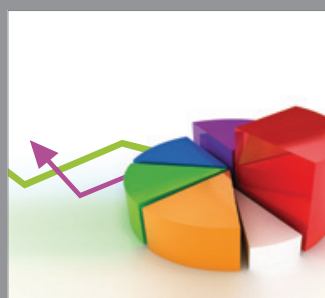

ournal of

Probability and Statistics

Promensencen
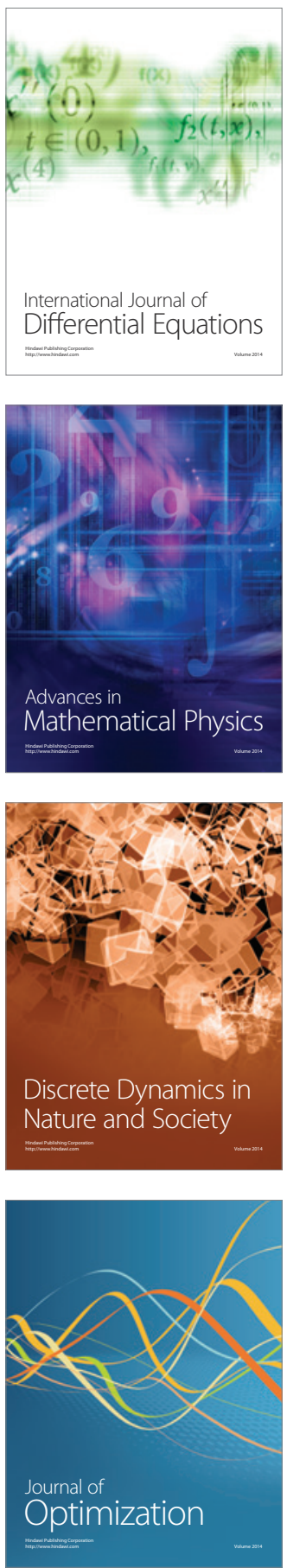